\section{Shared data are key to beating threat from flu}

SIR - We fully support Ilaria Capua in her call for avian-influenza researchers to release data to the public, rather than store them in restricted databases, as reported in your Editorial "Dreams of flu data" (Nature 440, 255-256; 2006). Keeping sequences secret, whatever the motivation, slows down scientific progress and hinders efforts to protect public health. The influenza genome sequencing project (www.niaid.nih.gov/ $\mathrm{dmid} / \mathrm{genomes} / \mathrm{mscs} /$ influenza.htm) has, in the past year, sequenced more than 1,000 complete genomes of human influenza and released them to GenBank (www.ncbi.nih. gov/Genbank). All sequences are deposited immediately they are completed, as agreed by all the centres contributing samples to this project. We believe unrestricted access to these data will jump-start research in many influenza labs across the globe, advancing vaccine design and enhancing our understanding of the virus.

We call on all other scientists who might be sitting on influenza-virus data, whether human or animal data, to follow this example. We also join Capua and Nature in calling for the World Health Organization and the US Centers for Disease Control and Prevention to make future - and archived data available to the scientific community. It is time for the community of influenza researchers to recognize, as the human genome sequencing project did ten years ago, that immediate public release of sequence data provides the greatest benefits to human health. The influenza virus does not respect national or other artificial boundaries, and we all need to work together to control it. Steven Salzberg ${ }^{\star}$, Elodie Ghedin $\dagger$, David Spirot *Center for Bioinformatics and Computational Biology, University of Maryland, College Park, Maryland 20742, USA

†The Institute for Genomic Research, 9712 Medical Center Drive, Rockville, Maryland 20850, USA

\section{Dogs can play useful role as sentinel hosts for disease}

\footnotetext{
SIR — News that Thai dogs have tested positive for antibodies to the influenza $A$ $\mathrm{H} 5 \mathrm{~N} 1$ virus ( ${ }^{\alpha} \mathrm{Thai}$ dogs carry bird-flu virus, but will they spread it? ${ }^{m}$ Nature 439,773 ; 2006) reinforces our notion that carnivore and scavenger species have the potential to act as important sentinel hosts for emerging human and livestock diseases, providing a valuable tool for surveillance and for determining spatial and temporal patterns of infection.

Domestic dogs may prove particularly
}

useful as sentinel hosts, especially in developing countries. They are ubiquitous, with one dog for every 7 to 21 people in most parts of Africa and Asia. Dogs are known to be susceptible to a wide range of emerging human infections, and, as freeroaming scavengers in many parts of the world, they effectively 'sample' widely from a community environment. Despite appearances, domestic dogs in most developing countries are generally accessible for safe handling and sampling.

Our experience in Africa and Asia suggests that sampling dogs for disease surveillance would be particularly cost-effective if carried out in combination with rabies vaccination campaigns, as this provides owners with a strong incentive to participate. During these campaigns, several hundred dogs per day could be accessible for sampling at a cost of US\$1-2 per dog vaccinated (K. Bögel and F. X. Meslin Bull. World Health Organ. $68,281-291 ; 1990$ ).

Domestic dogs, like other carnivore and scavenger species, may act as 'bioaccumulators' of pathogen exposure, with consumption of infected host material resulting in high rates of seroconversion. We suggest that they could therefore usefully be induded as part of surveillance strategies to increase the efficiency of pathogen detection, particularly for pathogens that occur at low prevalences in animal reservoirs or are maintained in wild animal populations that are difficult to sample.

Age-seroprevalence data can also allow timing of outbreaks to be established retrospectively and with reasonable accuracy, for at least a number of years. This would be particularly valuable in areas where reporting and laboratory confirmation of human and animal disease outbreaks are limited, which may apply in many parts of the developing world.

Sarah Cleaveland ${ }^{\star}$, Francois X. Meslin $\dagger$, RobertBreimant

*Wildife and Emerging Disease Section, Royal (Dick)School of Veterinary Studies, University of Edinburgh, Easter Bush, Roslin, Midlothian EH25 9RG, UK

†Department of Food Safety, Zoonoses and Foodborne Diseases, World Health Organization, 1211 Geneva 27, Switzerland † International Emerging Infections Program, Centers for Disease Control and Prevention, PO Box 606, Village Market, Gigiri, Nairobi, Kenya

\section{Ecological society supports its African counterparts}

SIR - Your Editorial "It's academic" (Nature $439,762-764 ; 2006)$ reports the need for stronger national academies in Africa. The British Ecological Society launched its Building Capacity for Ecology Fund in
January 2006, to support the development of ecological societies in Africa and Eastern Europe. The society has put $£ 500,000$ (US $\$ 70,000$ ) of its own money into this exciting initiative. As president (J.H.L.) and past-president (A.H.F.) of the society, we hope that others will join us in this or similar initiatives, as it is vital that the science of ecology is promoted and used by policymakers in Africa.

John H. Lawton, Alastair H. Fitter

British Ecological Society, 26 Blades Court, Deodar Road, London SW15 2NU, UK

\section{Populations who test drugs should benefit from them}

SIR — Paul Herrling, in his Commentary article "Experiments in social responsibility" (Nature 439, 267-268; 2006), describes pharmaceutical companies moving towards a more progressive approach to drug development and distribution in poor countries. But it is important to note that, even when research in developing nations leads to effective treatments, there is still the danger of local populations being exploited.

Recent dinical trials of a hepatitis Evaccine in Nepal are a case in point. Run by GlaxoSmithKline and the US government Armed Forces Research Institute of Medical Sciences, the trials showed an impressive on-treatment efficacy for the experimental vaccine (see M. P. Shrestha and R. N. Scott's report to the American Society for Tropical Medicine and Hygiene at www.astmh.org/meetings_new/ ASTMH_05_FP2.pdf). But the methodology of the trial raises questions about the ethics of clinical-trial conduct among vulnerable populations. The research team had to drop original plans to test civilian volunteers in the city of Lalitpur, after local people objected to a lack of informed consent or participation in trial design (for details of these events, see J. Andrews Am. J. Bioethics 5, W1; 2005). Instead, they gave the experimental vaccine to soldiers in the Royal Nepalese Army, who are vulnerable as members of the armed forces and as some of the poorest people in a least-developed' country.

These ethical issues take on greater importance now that the hepatitis $E$ vaccine may have public-health usefulness. Will the Nepalese community benefit? Or will the results be used only to develop a profitable vaccine for (mainly Western) travellers and US soldiers? We hope that GlaxoSmithKline and its collaborators make this vaccine accessible to the populations placed at risk by the trial, in line with the well-intentioned humanitarianism that Herrling describes. Sanjay Basu, Jason Andrews, DuncanSmith-Rohrberg Yale University School of Medicine, 129 York Street, New Haven, Connecticut 06511, USA 